\title{
Kindlin-2 siRNA inhibits vascular smooth muscle cell proliferation, migration and intimal hyperplasia via Wnt signaling
}

\author{
XIAOLIN WU ${ }^{1,2^{*}}$, WENWEI LIU ${ }^{2 *}$, HONG JIANG ${ }^{1}$, JING CHEN $^{1}$, JICHUN WANG $^{1}$, RUI ZHU ${ }^{2}$ and BIN LI $^{2}$ \\ ${ }^{1}$ Department of Cardiology, Renmin Hospital of Wuhan University, Wuhan, Hubei 430060; ${ }^{2}$ Department of Cardiology, \\ Xiangyang Central Hospital, Affiliated Hospital of Hubei University of Arts and Science, Xiangyang, Hubei 441000, P.R. China
}

Received May 14, 2015; Accepted December 1, 2015

DOI: $10.3892 / \mathrm{ijmm} .2015 .2429$

\begin{abstract}
It is known that vascular smooth muscle cell (VSMC) proliferation and migration leads to intimal hyperplasia in cases of atherosclerosis and restenosis. In the present study, we investigated the effects of kindlin-2 on VSMC proliferation, migration and intimal hyperplasia, and the underlying mechanisms. The left common carotid artery of Sprague-Dawley rats were subjected to balloon injury in order to induce intimal hyperplasia, and then transfected with kindlin- 2 small interfering RNA (siRNA) lentivirus or negative control siRNA lentivirus. We noted that the degree of intimal hyperplasia 4 weeks after balloon injury was significantly reduced in arteries transfected with kindlin-2 siRNA lentivirus $(\mathrm{P}<0.05)$. In vitro, kindlin-2 siRNA suppressed VSMC proliferation and migration induced by Wnt3a (100 ng/ml). Western blot analyses and RT-qPCR revealed that kindlin-2 regulated $W n t / \beta$-catenin signaling and thereby modulated the expression of $\beta$-catenin target genes, including c-myc and cyclin D1. This study demonstrated that kindlin-2 plays a critical role in VSMC proliferation, migration and intimal hyperplasia via Wnt signaling. Therefore, blocking the activity of kindlin-2 represents a novel therapeutic strategy for vascular injury.
\end{abstract}

\section{Introduction}

Vascular smooth muscle cell (VSMC) proliferation, migration and cell-extracellular matrix (ECM) adhesion are all known to contribute to the development of intimal hyperplasia in certain vascular pathologies, including restenosis and atherosclerosis $(1,2)$. Interactions between VSMCs and the ECM regulate these processes through binding of the integrin family of cell adhesion receptors (2). A key mediator of integrin signaling

Correspondence to: Dr Hong Jiang, Department of Cardiology, Renmin Hospital of Wuhan University, 99 Zhang Zhidong Road, Wuhan, Hubei 430060, P.R. China

E-mail: jiang_hongw@aliyun.com

${ }^{*}$ Contributed equally

Key words: kindlin-2, vascular smooth muscle cell, proliferation, migration, intimal hyperplasia is kindlin-2, a recently discovered family of evolutionarily conserved four point one protein, ezrin, radixin and moesin (FERM) domain-containing proteins that binds directly to the cytoplasmic tails of $\beta 1$ and $\beta 3$ integrins (3-5). Kindlin-2 is essential for integrin clustering and activation, and regulates cell adhesion and directed migration by guiding the formation and maturation of focal adhesions (FA) and the organization of the cytoskeleton (6). Due to its essential role in integrin activation, kindlin-2 is involved in many important physiological processes, including heart development, cell migration and cancer progression $(4,7)$. In mice, the loss of kindlin- 2 causes peri-implantation lethality resulting from detachment of the endoderm and epiblast from the basement membrane as a consequence of diminished levels of $\beta 1$-integrin and also of $\beta 1$-integrin activation (8). In zebrafish, knockdown of the kindlin-2 gene resulted in severe abnormalities in the development of the heart. Ultrastructural analysis has revealed disrupted intercalated disc formation and that myofibrils failed to attach to the membrane complexes (9). Even partial inactivation of the kindlin-2 gene markedly impairs angiogenesis in mice and zebrafish, which arises from defective activation of integrin $\alpha \mathrm{V} \beta 3$ (10). In in vitro experiments using cells derived from kindlin-2 deficient mice or small interfering RNA (siRNA)-knockdown mice, defects in integrin activation despite the presence of talin were noted (10). Recently, Yu et al found that kindlin-2 forms a transcriptional complex with $\beta$-catenin and transcription factor 4 (TCF4) and enhances Wnt signaling $(11,12)$.

Wnts are a family of secreted glycoproteins that bind to transmembrane Frizzled receptors and initiate signaling cascades, and it has been previously noted that they play indispensable roles during cell proliferation, migration, adhesion and survival (13-15). Activation of the Wnt/ $\beta$-catenin signaling pathway leads to $\beta$-catenin nuclear translocation and complex formation with lymphoid enhancer-binding factor/T cell factor (LEF/TCF) transcription factors, followed by transcriptional activation of target genes in the nucleus (13-15). In previous studies using animal models of intimal hyperplasia, increased $\beta$-catenin levels have been noted, and the role of Wnt signaling in VSMCs has thus been considered $(16,17)$. Wnt signaling is a novel regulator of VSMC proliferation and, thereby, intimal hyperplasia (17). These findings suggest that kindlin-2 and Wnts have overlapping functions in regulating cell behavior and physiological processes. However, kindlin-2-mediated 
interactions are not yet fully understood, and the relationship between kindlin-2 and Wnt signaling remains to be fully elucidated. Moreover, little is known about the role kindlin-2 plays in VSMC proliferation, migration and intimal hyperplasia.

In the present study, we used RNA interference (RNAi) to examine the effects of kindlin-2 on recombinant Wnt3a-induced VSMC proliferation and migration in vitro and intimal hyperplasia following balloon catheter injury of the carotid artery of rats in vivo.

\section{Materials and methods}

Construction and production of lentiviral vectors. Three siRNA sequences targeting rat kindlin-2 (GenBank accession no., NM_001011915) and a negative control sequence were constructed by Genechem (Shanghai, China). The kindlin-2 siRNA sequence which performed best was CAAACA GATAACAGCACGG, and that of negative control siRNA (NC siRNA) was TTCTCCGAACGTGTCACGT (data not shown). Short hairpin RNAs (shRNAs) were inserted into the lentiviral vector GV118 driven by the U6 promoter and containing the green fluorescent protein (GFP) reporter gene. All constructs were then verified by sequence analysis. Lentivirus-encoded shRNA against kindlin-2 and the control were produced by co-transfecting 293T cells (purchased from GeneChem, Shanghai, China) with Lipofectamine 2000 (Invitrogen, Carlsbad, CA, USA) according to standard protocols. The virus titers, measured in $293 \mathrm{~T}$ cells as transducing units/milliliter (TU/ml), were approximately $1 \times 10^{9} \mathrm{TU} / \mathrm{ml}$.

Rat model of carotid artery injury and lentiviral transfection. All animal protocols complied strictly with the Institutional Animal Care and Use Committee guidelines. The procedure for balloon injury in rat carotid arteries has been described previously (18). Briefly, male Sprague-Dawley (SD) rats $(n=45)$ (Wuhan University Experimental Animal Center, Wuhan, China), 3-4 months old and weighing 350-400 g were anesthetized by intraperitoneal injection of pentobarbital $(40 \mathrm{mg} / \mathrm{kg})$. After intravenous injection of $100 \mathrm{U} / \mathrm{kg}$ of heparin sodium, the left common, external and internal carotid arteries were exposed, and a balloon angioplasty catheter (balloon diameter $1.25 \mathrm{~mm}$, balloon length $15 \mathrm{~mm}$; Medtronic, Minneapolis, MN, USA) was introduced through the external carotid arteriotomy incision, advanced to the aortic arch, inflated to produce moderate resistance, and gradually withdrawn 3 times. For lentiviral transfection, a $50 \mu 1$ solution of kindlin-2 siRNA-GFP lentivirus $\left(2 \times 10^{8} \mathrm{TU} / \mathrm{ml}\right)$ or NC siRNA-GFP lentivirus $\left(2 \times 10^{8} \mathrm{TU} / \mathrm{ml}\right)$ was infused into the injured common carotid artery segment (approximately $20 \mathrm{~mm}$ in length) isolated by two microvascular clips, and incubated for $30 \mathrm{~min}$. The external carotid artery was then ligated, and blood flow through the common and internal carotid arteries was restored.

Hematoxylin and eosin $(H \& E)$ staining. The rats were sacrificed by jugular vein blood collection, which leads to massive loss of blood and death in rats, 4 weeks after balloon injury and lentiviral transfection, and the left common carotid arteries were harvested, fixed in $4 \%$ paraformaldehyde, and then embedded in paraffin. For harvesting the arteries, the rats were fixed on a board after being anesthetized. The left common carotid arteries were exposed by layer separation after skin incision, and the left common carotid arteries were then clipped after clamping the proximal and distal ends of the arteries. For morphologic analysis of intimal hyperplasia, five round crosssections ( $4 \mu \mathrm{m}$ thickness) were cut from the approximate middle of the artery, stained with $\mathrm{H} \& \mathrm{E}$, photographed, and analyzed using Image-Pro Plus 6.0 professional image analysis software (Media Cybernetics, Silver Spring, MD, USA). The intimal and medial cross-sectional areas of the carotid arteries were measured, and the intima/media ratios were also calculated.

VSMC culture and transfection. Primary VSMCs were isolated from the thoracic aortas of male SD rats $(100-150 \mathrm{~g}, \mathrm{n}=16)$. The rats were fixed on a board after being anesthetized. The thoracic arteries were exposed and clipped. The thoracic arteries were then placed in a dish with Dulbecco's modified Eagle's medium (DMEM) and the endothelial cells were removed by opthalmic tweezers. Finally, the remaining cells were the VSMCs. The cells were then cultured in DMEM containing $10 \%$ fetal bovine serum (FBS; HyClone, Logan, UT, USA), $100 \mathrm{U} / \mathrm{ml}$ penicillin, and $100 \mu \mathrm{g} / \mathrm{ml}$ streptomycin. The cells were incubated at $37^{\circ} \mathrm{C}$ in a humidified atmosphere of $95 \%$ air and $5 \% \mathrm{CO}_{2}$. The purity of the VSMCs was assessed at approximately $90 \%$ through studying the cell morphology and immunostaining with anti$\alpha$-actin antibody (A5228; Sigma, St. Louis, MO, USA). All VSMCs were used for experiments between the 3rd and 6th passages. VSMCs $\left(1 \times 10^{5}\right)$ were plated in 6-well plates and grown to approximately $50 \%$ confluence, then transfected with kindlin-2 siRNA lentivirus or NC siRNA lentivirus at multiplicities of transfection (MOI) of 100. Lentiviral transfection was validated by visualization of enhanced GFP under a fluorescence microscope (Nikon TE2000; Nikon, Tokyo, Japan).

Cell proliferation assay. Cell proliferation was measured by studying the incorporation of bromodeoxyuridine (BrdU) during DNA synthesis in proliferating cells. Untreated VSMCs were seeded at a density of 5,000 cells/well in 96-well culture plates in DMEM with $10 \%$ FBS and cultured for $24 \mathrm{~h}$. VSMCs were then starved in DMEM without FBS for $12 \mathrm{~h}$ to achieve synchronous growth, and transfected with kindlin-2 siRNA lentivirus or NC siRNA lentivirus at MOI of 100 for $12 \mathrm{~h}$. Wnt3a (final concentration, $100 \mathrm{ng} / \mathrm{ml}$; R\&D Systems, Minneapolis, MN, USA) was added to each well after transfection, and $48 \mathrm{~h}$ after transfection $20 \mu \mathrm{l}$ BrdU was also added to each well to label the cells during $24 \mathrm{~h}$ of incubation. Quantification of BrdU incorporation was performed using a BrdU cell proliferation assay kit (Millipore, Billerica, MA, USA) according to the manufacturer's instructions. The absorbance was read at a wavelength of $450 \mathrm{~nm}$ with a spectrophotometric plate reader (Infinite M200 PRO; Tecan, Männedorf, Switzerland).

Cell migration assay. The VSMC migration assay was performed using Transwell cell culture inserts (Corning, High Wycombe, UK) in 24-well plates. One hundred microliters of VSMCs, which were stably transfected with kindlin-2 siRNA, NC siRNA or left untreated $\left(3 \times 10^{5}\right.$ cells $\left./ \mathrm{ml}\right)$, suspended in serum-free DMEM, were added to the upper polycarbonate membrane insert (pore size, $8 \mu \mathrm{m}$ ). Wnt3a (final concentration, $100 \mathrm{ng} / \mathrm{ml}$ ) was added to the upper chamber, and $600 \mu \mathrm{l}$ culture medium containing $10 \%$ FBS was added to the lower chamber. 
The cells were allowed to migrate for $24 \mathrm{~h}$ while the plates were incubated in a humidified incubator in an atmosphere with $5 \% \mathrm{CO}_{2}$ at $37^{\circ} \mathrm{C}$. After $24 \mathrm{~h}$, the cells that remained on the upper surface of the membrane were removed with a cotton swab. The membrane was fixed with anhydrous methanol for $20 \mathrm{~min}$ at room temperature and then stained with $0.1 \%$ crystal violet for $15 \mathrm{~min}$. A microscope was used to determine the number of migratory cells by counting the cells in 5 randomly selected fields of view. All experiments were performed in triplicate.

Reverse transcription-quantitative PCR (RT-qPCR). After transfection and Wnt3a (100 ng/ml) stimulation for 3 days, total RNA was isolated from VSMCs using TRIzol (Invitrogen) reagent. For RT-qPCR, we performed reverse transcription to produce cDNA from total RNA with oligo(dT), and the fragments were then amplified with a SYBR-Green-based assay kit (Invitrogen) according to the manufacturer's instructions. Thermal cycling conditions comprised an initial denaturation step at $50^{\circ} \mathrm{C}$ for $2 \mathrm{~min}, 95^{\circ} \mathrm{C}$ for $10 \mathrm{~min}$, followed by 40 cycles $\left(95^{\circ} \mathrm{C}\right.$ for $30 \mathrm{sec} ; 60^{\circ} \mathrm{C}$ for $\left.30 \mathrm{sec}\right)$. $\beta$-actin was used for normalization, and the data were analyzed using the $2^{-\Delta \Delta \mathrm{Ct}}$ method. The primers were as follows: kindlin-2 forward, 5'-AGATCT GGCTTCGCTGTGAT-3' and reverse, 5'-CGGGATTGATG TCAGTTGTG-3'; c-myc forward, 5'-CGAGCTGAAGCGTAG CTTTT-3' and reverse, 5'-CTCGCCGTTTCCTCAGTAAG-3'; cyclin D1 forward, 5'-GCGTACCCTGACACCAATCT-3' and reverse, 5'-GGCTCCAGAGACAAGAAACG-3'; $\beta$-actin forward, 5'-CACGATGGAGGGGCCGGACTCATC-3' and reverse, 5'-TAAAGACCTCTATGCCAACACAGT-3'.

Western blot analysis. After transfection, VSMCs treated with Wnt3a (100 ng/ml) and PBS (control) for 3 days were harvested. The cells were lysed with RIPA buffer supplemented with proteinase inhibitors for $30 \mathrm{~min}$ on ice. Protein concentration was measured with a BCA protein assay (Pierce, Rockford, IL, USA). Proteins were then separated by SDS-polyacrylamide gels and transferred to nitrocellulose membranes. The membranes were blocked with $10 \%$ non-fat dry milk, and then immunoblotted overnight at $4{ }^{\circ} \mathrm{C}$ with antibodies that recognize kindlin-2 (1:500; K3269; Sigma), $\beta 1$-integrin (1:500; 04-1109; Millipore), total $\beta$-catenin $(1: 1,000$; 9562), phospho$\beta$-catenin (Ser675, 1:1,000; 9567) (both from Cell Signaling Technology, Beverly, MA, USA), total glycogen synthase kinase-3 $\beta$ (GSK-3 $\beta$, 1:400; Sc-9166), phospho-GSK-3 $\beta$ (Ser9, 1:500; Sc-373800) and $\beta$-actin $(1: 1,000 ;$ Sc-32251) (all from Santa Cruz Biotechnology, Inc., Santa Cruz, CA, USA). After three washes, the blots were incubated with peroxidaseconjugated secondary antibodies (Pierce) for $1 \mathrm{~h}$ at room temperature, and subsequently analyzed by an ECL detection system (Beijing Liuyi Instrument Factory, Beijing, China).

Flow cytometric analysis. VSMCs were transfected with lentiviruses of kindlin-2 siRNA or NC siRNA and supplemented with Wnt3a (100 ng/ml) for 3 days. $\beta 1$-integrin expression on the VSMC surface was evaluated by indirect immunofluorescence using flow cytometry. After being rinsed in PBS, the cells were incubated with rabbit anti-rat antibody against $\beta 1$-integrin $(1: 70)$ and mouse anti-rat antibody against active $\beta 1$-integrin (1:200; MAB2079Z) (both from Millipore) for $30 \mathrm{~min}$ at room temperature in the dark. The cells were then washed again and incubated with phycoerythrin (PE)-conjugated goat anti-rabbit secondary IgG (1:50; bs-0295G) and Cy3-conjugated goat anti-mouse secondary IgG (1:50; bs-0296G) (both from Bioss, Beijing, China) for $45 \mathrm{~min}$ and analyzed by flow cytometry using Becton-Dickinson FACSCalibur and CellQuest software.

Statistical analysis. All statistical analysis was performed with Statistical Product and Service Solutions 17.0 software (SPSS 17.0). Data are presented as the means \pm SEM. All values were analyzed using the Student's t-test for comparisons between two groups or one-way ANOVA for multiple comparisons. A P-value $<0.05$ was considered to indicate a statistically significant difference.

\section{Results}

Kindlin-2 RNAi suppresses intimal hyperplasia. Four weeks after balloon injury and lentiviral transfection, the degree of intimal hyperplasia was evaluated morphologically and quantitatively (Fig. 1). Kindlin-2 siRNA lentivirus treatment significantly reduced intimal hyperplasia $(\mathrm{P}<0.05)$, and the intima/media ratio was also markedly lower in kindlin-2 siRNA lentivirus-transfected arteries $(0.687 \pm 0.117)$ than in NC siRNA lentivirus-transfected arteries $(1.545 \pm 0.277)(\mathrm{P}<0.05)$.

Kindlin-2 RNAi attenuates the VSMC proliferation induced by Wnt3a. As shown in Fig. 2C, kindlin-2 mRNA expression was dramatically reduced in VSMCs transfected with kindlin-2 siRNA lentivirus $(\mathrm{P}<0.05)$, but not in VSMCs transfected with NC siRNA lentivirus $(\mathrm{P}>0.05)$. Compared with the control group, a $47 \%$ reduction of kindlin-2 mRNA was observed in VSMCs transfected with kindlin-2 siRNA lentivirus $(\mathrm{P}<0.05)$, indicating that kindlin-2 RNAi is effective. Subsequently, we examined the effect of kindlin-2 RNAi on Wnt3a-induced VSMC proliferation by measuring the nuclear incorporation of BrdU (DNA synthesis) and the mRNA expression levels of c-myc and cyclin D1, which are critical genes involved in cell cycle progression and cell proliferation; Wnt3a is a prominent member of the Wnt family and can activate the canonical Wnt pathway and induce cell proliferation $(19,20)$. We observed that kindlin-2 RNAi resulted in significant inhibition of BrdU incorporation compared with the control group and the NC siRNA group with or without Wnt3a stimulation $(\mathrm{P}<0.05$; Fig. $2 \mathrm{~A})$. The c-myc and cyclin D1 mRNA expression levels were significantly suppressed by kindlin-2 siRNA lentivirus at MOI of $100(\mathrm{P}<0.05$; Fig. 2B and D). NC siRNA lentivirus, which encodes for a nonhomologous shRNA, did not affect c-myc and cyclin D1 mRNA expression. After exposure of VSMCs to Wnt3a at a concentration of $100 \mathrm{ng} / \mathrm{ml}$ for 3 days, the levels of c-myc and cyclin D1 mRNA were significantly upregulated by 1.9 - and 2.3-fold, respectively $(\mathrm{P}<0.05)$. By contrast, Wnt3a-induced expression of c-myc and cyclin D1 was also markedly inhibited by pretreatment with kindlin-2 siRNA lentivirus $(\mathrm{P}<0.05)$.

Kindlin- 2 regulates cell proliferation by Wnt/ $\beta$-catenin signaling. $\beta$-catenin and GSK-3 $\beta$ were examined in this study, as they are considered to be the major downstream Wnt signaling molecules (13-15). Kindlin-2 has been found to be coexpressed with $\beta$-catenin in the invasive front of tumors and in tumor cells (11). To understand the mechanisms 


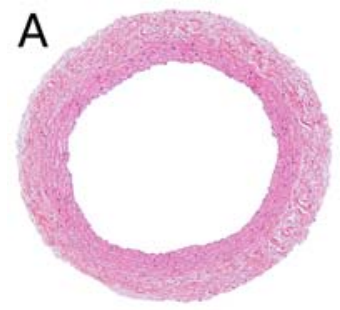

Control

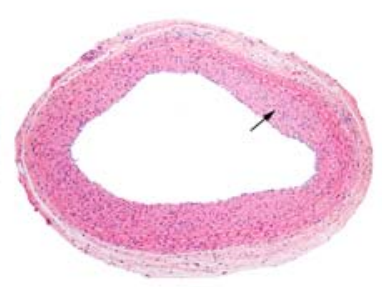

NC siRNA

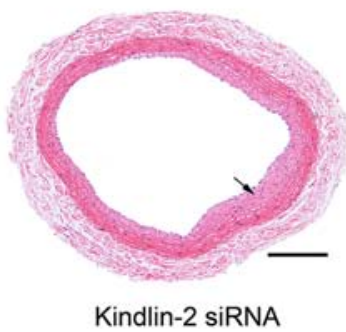

Kindlin-2 siRNA

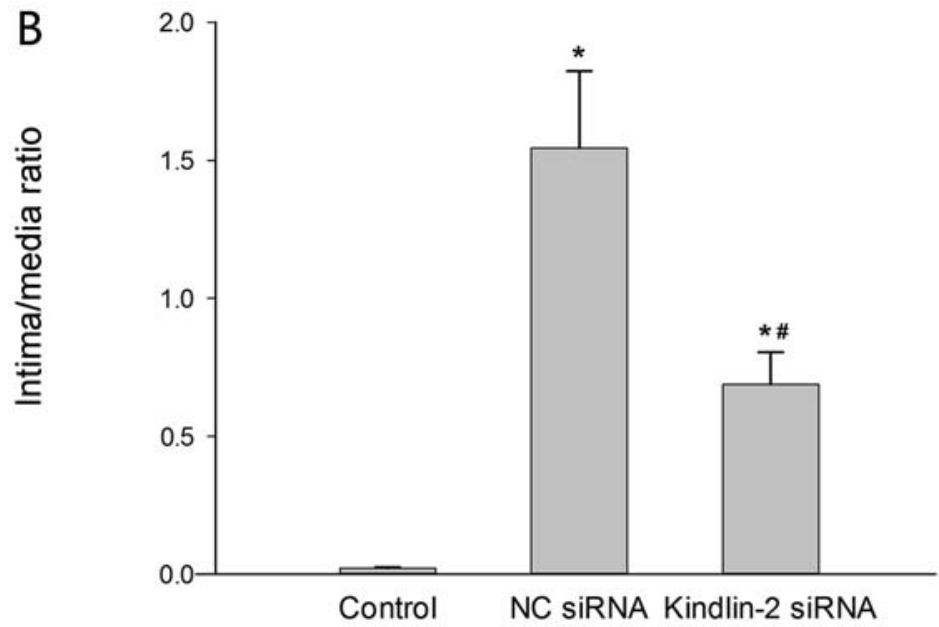

Figure 1. Effects of kindlin-2 silencing on intimal formation in balloon-injured carotid arteries. (A) Representative histologic sections of hematoxylin and eosin-stained carotid arteries. Scale bar represents $100 \mu \mathrm{m}$. (B) Quantitative analysis of ratio of intima to media was calculated 4 weeks after balloon injury and lentiviral transfection. ${ }^{~} \mathrm{P}<0.05$ vs. control group; ${ }^{*} \mathrm{P}<0.05$ vs. negative control (NC) siRNA group.
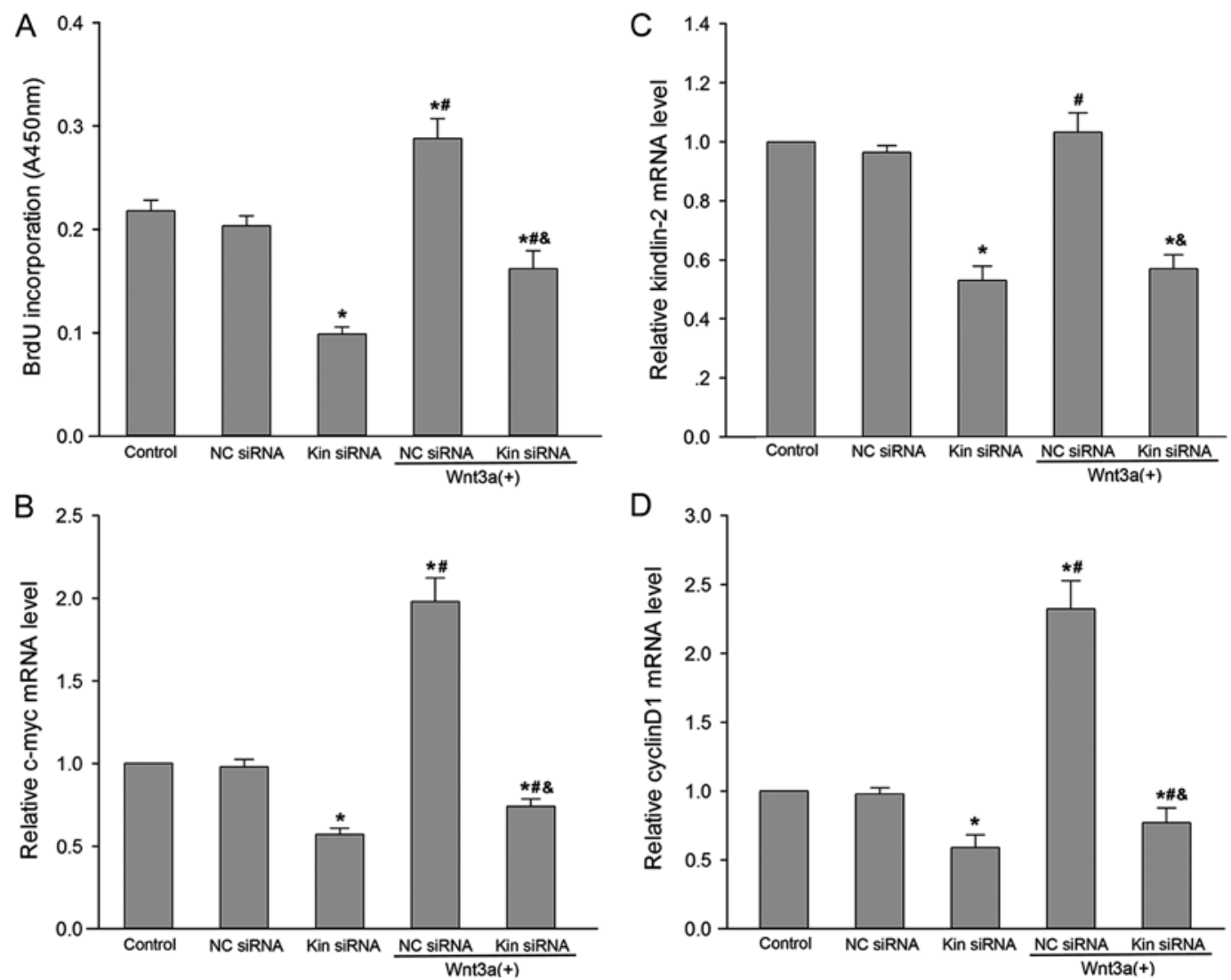

Figure 2. Inhibitory effect of kindlin-2 RNAi on Wnt3a-induced vascular smooth muscle cell (VSMC) proliferation. VSMCs were stimulated for 3 days with recombinant Wnt3a (100 ng/ml) after lentiviral transfection. Cell proliferation was evaluated by (A) BrdU incorporation assay and (B) studying the c-myc, (C) kindlin-2 and (D) cyclin D1 mRNA expression levels. Kindlin-2 siRNA (Kin siRNA) transfection of VSMCs resulted in a significant inhibition of BrdU incorporation. RT-qPCR indicated the efficient suppression of kindlin-2, c-myc and cyclin D1 mRNA in Wnt3a- and kindlin-2 siRNA-treated VSMCs. ${ }^{*}<0.05$ vs. control group and negative control (NC) siRNA group; ${ }^{\text {}} \mathrm{P}<0.05$ vs. kindlin-2 siRNA group; ${ }^{\text {\& }} \mathrm{P}<0.05$ vs. NC siRNA + Wnt $3 a$ group. 

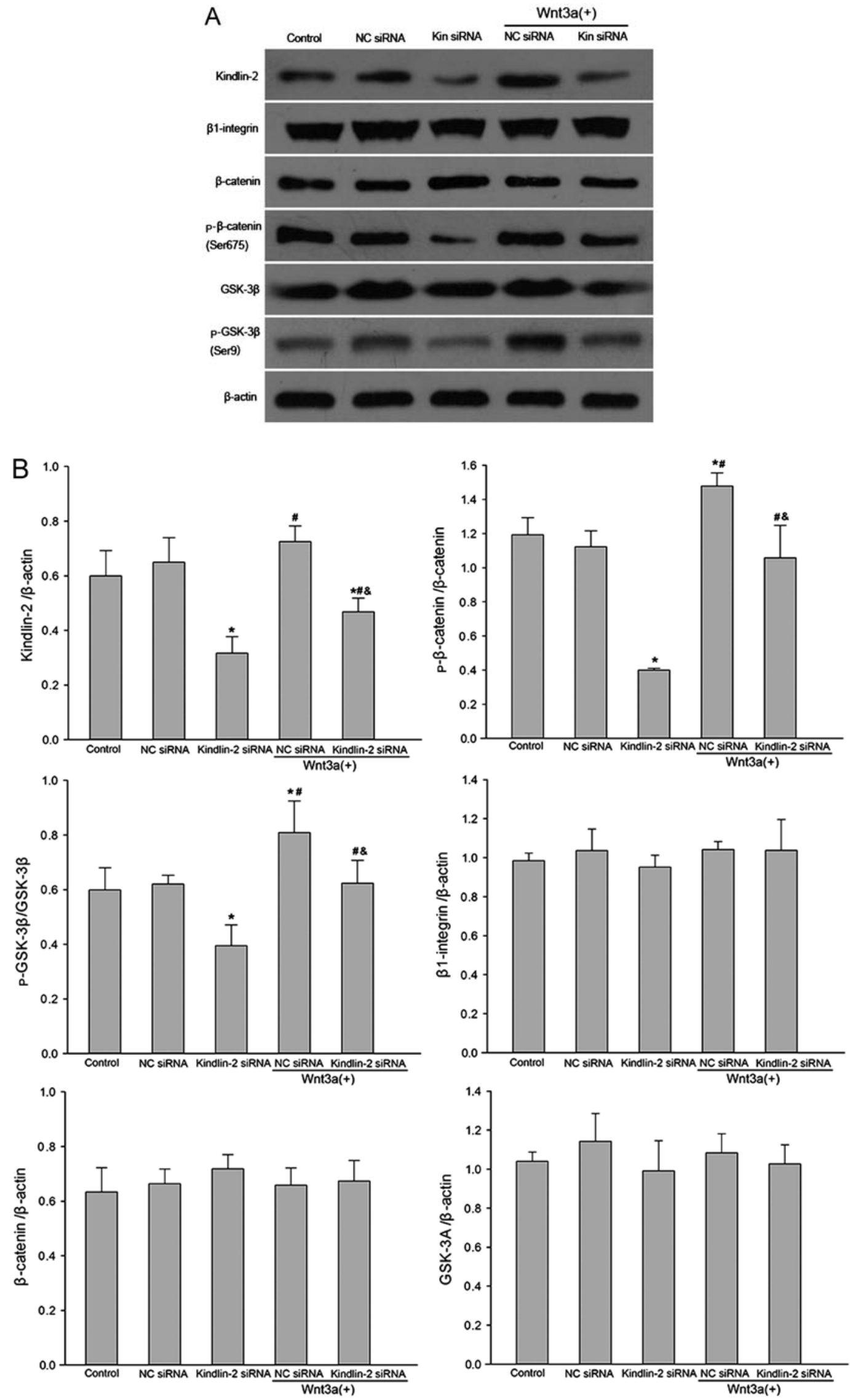

Figure 3. Kindlin-2 knockdown suppresses cell proliferation via Wnt/ $\beta$-catenin signaling. Vascular smooth muscle cells (VSMCs) were treated with Wnt3a $(100 \mathrm{ng} / \mathrm{ml})$ for 3 days after lentiviral transfection. (A) Protein expression of kindlin-2, $\beta 1$-integrin, total $\beta$-catenin, $\mathrm{p}$ - $\beta$-catenin (Ser675), total glycogen

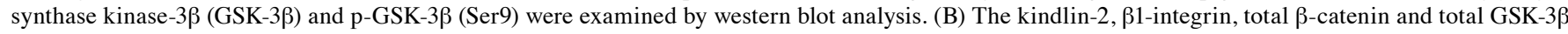
protein signals were quantified and normalized to that of $\beta$-actin. The $p$ - $\beta$-catenin and p-GSK-3 $\beta$ protein signals were normalized to those of total $\beta$-catenin and total GSK- $3 \beta$, respectively. ${ }^{\mathrm{P}}<0.05$ vs. control group and negative control $(\mathrm{NC})$ siRNA group; ${ }^{*} \mathrm{P}<0.05$ vs. kindlin- 2 siRNA group; ${ }^{\&} \mathrm{P}<0.05$ vs. NC siRNA + Wnt3a group. 
A

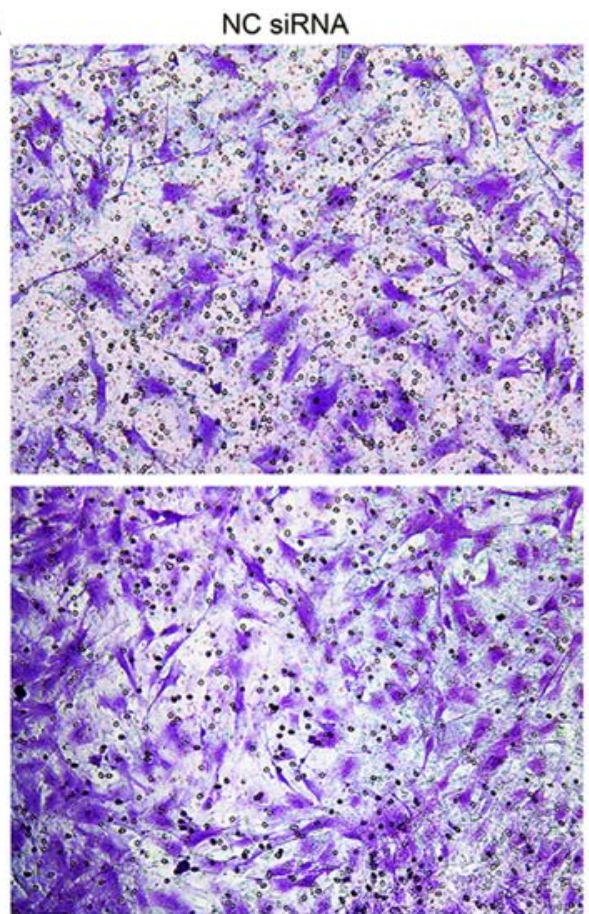

NC siRNA+Wnt3a

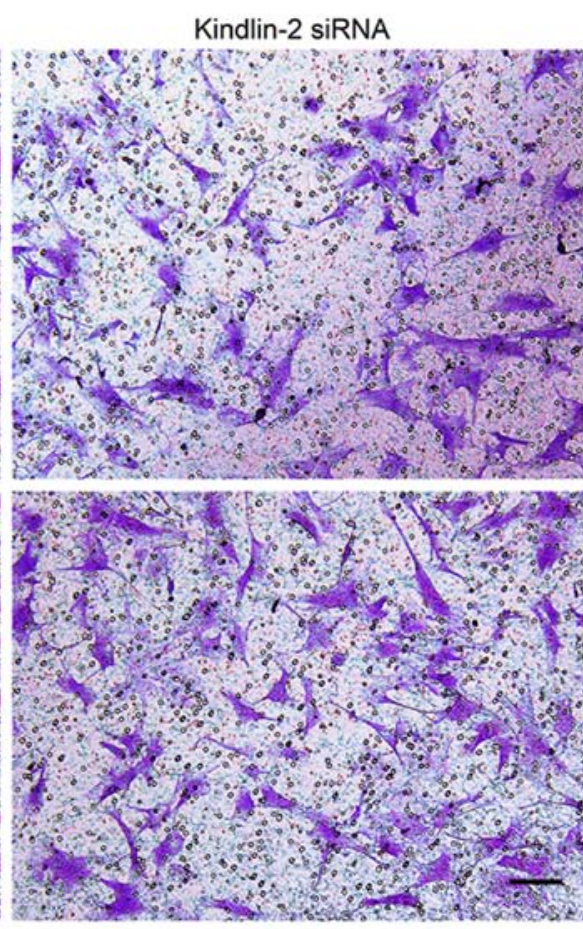

Kindlin-2 siRNA+Wnt3a

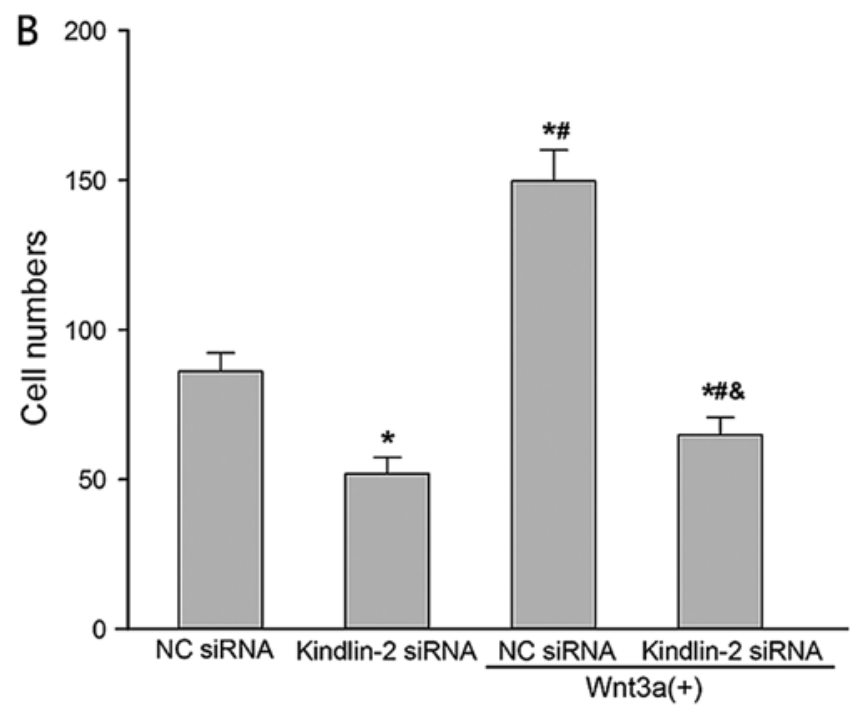

Figure 4. Kindlin-2 RNAi impairs vascular smooth muscle cell (VSMC) migration. (A) VSMCs on Transwell inserts were stained with $0.1 \%$ crystal violet (x200). Scale bar, $100 \mu \mathrm{m}$. (B) The number of migratory VSMCs transfected with kindlin-2 siRNA lentivirus was significantly lower than that of negative control (NC) siRNA group and Wnt3a treatment group. ${ }^{*} \mathrm{P}<0.05$ vs. NC siRNA group; ${ }^{*} \mathrm{P}<0.05$ vs. kindlin-2 siRNA group; ${ }^{\circ} \mathrm{P}<0.05$ vs. NC siRNA + Wnt3a group.

of kindlin-2 knockdown on VSMC proliferation induced by Wnt3a, we analyzed the protein expression of kindlin-2, $\beta 1$-integrin, $\beta$-catenin and GSK-3 $\beta$ (Fig. 3). Our results showed that kindlin-2 knockdown significantly decreased protein expression levels of kindlin-2, p- $\beta$-catenin (Ser675) and p-GSK-3 $\beta$ (Ser9) $(\mathrm{P}<0.05)$. Treatment of VSMCs with Wnt3a upregulated the expression of kindlin-2, $\mathrm{p}-\beta$-catenin (Ser675) and $\mathrm{p}-\mathrm{GSK}-3 \beta$ (Ser9) $(\mathrm{P}<0.05)$. However, the expression of $\beta 1$-integrin, total $\beta$-catenin and total GSK-3 $\beta$ did not differ significantly between groups $(\mathrm{P}>0.05)$.

Kindlin-2 depletion impairs VSMC migration. We performed a Transwell migration assay to investigate the effect of kindlin-2 RNAi and Wnt3a treatment on VSMC migration. The migratory ability of VSMCs transfected with kindlin-2 siRNA lentivirus was significantly decreased ( $\mathrm{P}<0.05$; Fig. 4). After treatment with Wnt3a, the migratory ability of VSMCs was significantly increased $(\mathrm{P}<0.05)$. The number of cells that migrated across the polycarbonate membrane was higher in the NC siRNA group and Wnt3a treatment group than in the kindlin-2 siRNA group $(\mathrm{P}<0.05)$.

Kindlin-2 and Wnt 3 a regulate integrin activation. Since kindlin-2 knockdown and treatment with Wnt3a did not markedly affect the protein expression of $\beta 1$-integrin, we quantified the expression of active $\beta 1$-integrin on the surface of VSMCs 


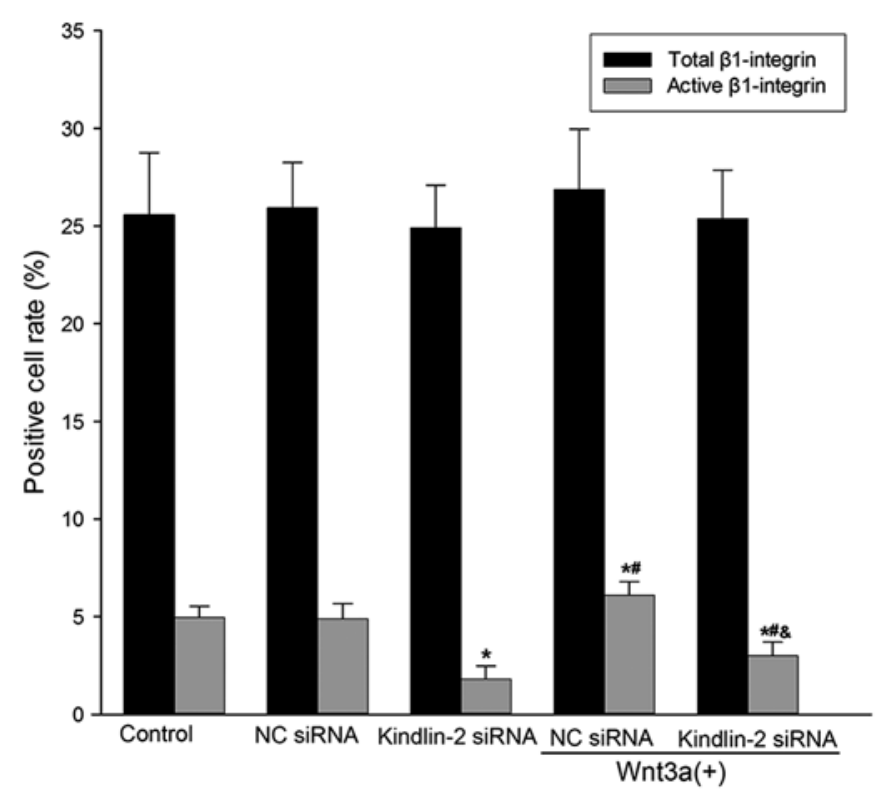

Figure 5. Kindlin-2 and Wnt3a regulate $\beta 1$-integrin activation. Active $\beta 1$-integrin and total $\beta 1$-integrin expression on the surface of vascular smooth muscle cells (VSMCs) was evaluated by flow cytometry. Kindlin-2 knockdown significantly reduced the active- $\beta 1$-integrin levels on the VSMC surface. Treatment with Wnt3a activated $\beta 1$-integrin but did not markedly affect the expression levels on the cell surface. ${ }^{*} \mathrm{P}<0.05$ vs. control group and negative control (NC) siRNA group; ${ }^{~} \mathrm{P}<0.05$ vs. kindlin- 2 siRNA group; ${ }^{\&} \mathrm{P}<0.05$ vs. NC siRNA + Wnt3a group.

using flow cytometry. The anti-active- $\beta 1$-integrin antibody is specific for the active conformation of rat $\beta 1$-integrin, and it can also discriminate between the different activated states. Therefore, it is exceptionally useful for studying how $\beta 1$-integrin activation is regulated. Our results showed that knockdown of kindlin-2 significantly reduced the VSMC surface levels of active- $\beta 1$-integrin $(\mathrm{P}<0.05$; Fig. 5$)$. VSMCs that were stimulated with Wnt3a for 3 days after transfection bound to more active- $\beta 1$-integrin antibody than the kindlin-2 siRNA group $(\mathrm{P}<0.05)$. However, kindlin-2 knockdown or Wnt3a treatment did not have a marked effect on the total amount of $\beta 1$-integrin expression on the surface of VSMCs ( $>0.05$; Fig. 5). These results demonstrate that a minimal level of kindlin-2 is required for optimal integrin activation in VSMCs, and that Wnt3a treatment activates $\beta 1$-integrin without changing its expression levels.

\section{Discussion}

In the present study, to the best of our knowledge, we have provided the first direct evidence that kindlin-2 regulates VSMC proliferation and migration in vitro and intimal hyperplasia in vivo. Additionally, we have shown that $\mathrm{Wnt} / \beta$-catenin signaling is involved in signal transduction and the functional regulation of kindlin-2. Consequently, we propose that modification of kindlin-2 or Wnt signaling is a potential target for inhibition of VSMC proliferation, migration and intimal hyperplasia.

Kindlin-2 belongs to the kindlin family of proteins; the kindlins are emerging as a novel class of molecules which are implicated in integrin activation, a critical process for cell proliferation, migration, differentiation and adhesion as well as for cell-ECM interactions (7). They comprise three evolutionarily conserved members in vertebrates, kindlin-1, kindlin-2 and kindlin-3, which share considerable sequenctial and structural similarities (7,21-24). The kindlins have a FERM domain, which is interrupted by a pleckstrin homology domain, and bind directly to various classes of integrins as well as participating in inside-out integrin activation (7,21-25). Lossof-function mutations in kindlin-1 and kindlin-3 cause Kindler syndrome and leukocyte adhesion deficiency III syndrome, respectively, but no human disease has yet been associated with the kindlin-2 gene (7,22-24).

At present, limited information exists on the physiological functions of kindlin-2, and the data are mainly derived from knockout animal models and in vitro studies with cell lines. The essential role of kindlin-2 in development is demonstrated by its peri-implantational lethality and the abnormal heart development noted in kindlin-2 knockout mice and zebrafish $(8,9)$. At the cellular level, kindlin- $2^{-/-}$embryonic stem cells exhibited a normal proliferation rate, but strongly reduced adhesion to laminins and fibronectin was noted (8). In $\mathrm{C} 2 \mathrm{C} 12$ cells, kindlin-2 contributed to myocyte elongation and fusion in multinucleated myotubes (26). Knockdown of kindlin-2 in wild-type keratinocytes impaired cell spreading (27). Kindlin-2 is also important in the regulation of podocyte-matrix adhesion and matrix deposition (28). Our results revealed that kindlin-2 regulates VSMC proliferation and migration. However, in previous studies, overexpression and knockdown of kindlin-2 have yielded contradicting results regarding specific functions: evidence for both suppression of cancer cell invasion in leiomyosarcoma or colon carcinoma cell lines $(3,29)$ and stimulation of cell migration in fibroblasts, human umbilical vein endothelial cells (HUVECs), malignant mesothelioma and gastric cancer cells has been noted (6,30-32). These observations suggest that the functions of kindlin-2 are cell type- or integrin-specific, and that its role in cell motility differs depending on the biological context. For example, overexpression of kindlin-2 in Chinese hamster ovary (CHO) cells exogenously expressing $\alpha \mathrm{IIb} \beta 3$ integrin enhances its activation $(3,30)$. However, overexpression of kindlin-2 in the same type of cells inhibits endogenous $\alpha 5 \beta 1$-integrin activation (21). Taken together, these results suggest that alterations of kindlin-2 expression affect integrin-dependent functions. This study, and others, have observed that kindlin- 2 knockdown did not markedly affect the protein expression of $\beta 1$-integrin, but significantly reduced $\beta 1$-integrin activation $(6,28)$.

To date, kindlin-2 is the only kindlin protein that has not been implicated in disease pathophysiology, but it is rapidly emerging as a key molecule in cardiac and muscle development (7). Moreover, the role of kindlin-2 in cell proliferation and migration is not yet fully understood, and little is known about the signal transduction pathways of kindlin-2; determining these will be important in understanding the role of kindlin-2 in the pathophysiology of disorders of integrin activation. To determine the effect of kindlin-2 on cell proliferation and migration, in the present study, RNA-mediated interference experiments were performed on VSMCs. siRNAs targeting kindlin-2 or irrelevant RNA as a negative control were constructed and introduced to VSMCs, and kindlin-2 expression levels were analyzed by RT-qPCR and western blot analysis. Transfection with kindlin-2 siRNA but not NC siRNA significantly inhibited the expression of kindlin-2. We detected 
VSMC proliferation by measuring the nuclear incorporation of BrdU and VSMC migration using a Transwell assay. The results showed that kindlin-2 siRNA resulted in significant inhibition of BrdU incorporation compared with the NC siRNA group, and the migratory ability of VSMCs in the kindlin-2 siRNA group was significantly decreased.

Although these previous studies have suggested that kindlin-2 signaling is crucial to VSMC proliferation and migration, the underlying mechanism is still unknown. Previous studies have shown that kindlin-2 is important to the enhancement of $\mathrm{Wnt} / \beta$-catenin signaling, as it selectively binds to active $\beta$-catenin and stabilizes it by preventing GSK-3 $\beta$ (a negative regulator of Wnt signaling) from binding $(11,12)$. It is well known that the major downstream Wnt signaling target is $\beta$-catenin. Activation of the $\beta$-catenin signaling pathway occurs by inhibiting GSK-3 $\beta$ from phosphorylating the N-terminal part of $\beta$-catenin, which leads to rapid degradation of $\beta$-catenin. However, $\beta$-catenin is also activated by phosphorylation in the C-terminal at serine 675, which facilitates the translocation of $\beta$-catenin to the nucleus and enhances its transcriptional activity (20). In this study, we activated $\mathrm{Wnt} / \beta$-catenin signaling with recombinant Wnt3a to study the effect of kindlin-2 siRNA on VSMCs. We noted increased $\beta$-catenin phosphorylation at Ser675 and increased GSK-3 $\beta$ phosphorylation at Ser9 (inactivation of GSK-3 $\beta$ ) after Wnt3a stimulation. Kindlin-2 knockdown significantly decreased protein expression levels of $\mathrm{p}-\beta$-catenin (Ser675) and p-GSK-3 $\beta$ (Ser9). However, the expression of total $\beta$-catenin and GSK-3 $\beta$ was not markedly affected by kindlin-2 siRNA. Moreover, we noted that Wnt3a also elevated $\beta 1$-integrin activity and promoted VSMC migration.

Previous studies have revealed that $\mathrm{Wnt} / \beta$-catenin signaling is involved in the regulation of VSMC proliferation and migration (14-17,33). Wang et al showed that overexpression of constitutively active $\beta$-catenin increased cyclin D1 promoter activity in a rat VSMC line (34). Cyclin D1 protein is important for the regulation of the cell proliferation cycle. When cyclin D1 is amplified or expressed, the G1/S transition is shortened and cell proliferation is promoted; c-myc, which was investigated in the present study, is known to act as a proto-oncogene and cell proliferation-initiating factor (35). It has previously been pointed out that c-myc and cyclin D1 are downstream target genes of the $W n t / \beta$-catenin signaling pathway $(20,36)$. In the present study, we confirmed that treatment with $\mathrm{Wnt} 3$ a resulted in the activation of $\mathrm{Wnt} / \beta$-catenin signaling and increased the expression of $\beta$-catenin responsive genes c-myc and cyclin D1. Taken together, these results show that growth inhibition by kindlin-2 siRNA in VSMCs was manifested partly through the dysregulated nuclear translocation of $\beta$-catenin and the consequent downregulation of its transcriptional targets c-myc and cyclin D1.

VSMC proliferation and migration play a critical role in intimal hyperplasia through cellular expansion and ECM deposition (1). Elucidating the molecular mechanisms responsible for VSMC proliferation and migration has led to the development of novel therapeutic approaches. In this study, we have investigated how kindlin-2 silencing inhibits intimal hyperplasia. When we studied the vascular morphology in our study, we noted that intimal hyperplasia was clearly visible 4 weeks after balloon injury and that kindlin-2 siRNA lentivirus treat- ment significantly reduced intimal hyperplasia. Moreover, the intima/media ratio was also markedly reduced in arteries transfected with kindlin-2 siRNA lentivirus compared with arteries transfected with NC siRNA lentivirus. The possible reason for this result is that kindlin-2 RNAi inhibits VSMC proliferation and migration to the intima. Our data indicate that kindlin-2 knockdown plays dual roles in the treatment and prevention of intimal hyperplasia. Kindlin-2 siRNA not only inhibits VSMC proliferation and migration by Wnt signaling, but also suppresses VSMC migration by regulating $\beta$-integrin activation. Our results suggest that inhibition of kindlin-2 is an attractive therapeutic approach for prevention of intimal hyperplasia.

\section{Acknowledgements}

This study was supported by the National Science Foundation of China (NSFC) nos. 81170195 and 81200156 . We thank all teachers from the Renmin Hospital of Wuhan University for excellent technical assistance.

\section{References}

1. Marx SO, Totary-Jain H and Marks AR: Vascular smooth muscle cell proliferation in restenosis. Circ Cardiovasc Interv 4: 104-111, 2011.

2. Ho B, Hou G, Pickering JG, Hannigan G, Langille BL and Bendeck MP: Integrin-linked kinase in the vascular smooth muscle cell response to injury. Am J Pathol 173: 278-288, 2008.

3. Shi X, Ma YQ, Tu Y, Chen K, Wu S, Fukuda K, Qin J, Plow EF and Wu C: The MIG-2/integrin interaction strengthens cell-matrix adhesion and modulates cell motility. J Biol Chem 282: 20455-20466, 2007.

4. Khan AA, Shimokawa T, Strömblad S and Zhang H: Functional characterization of human kindlin-2 core promoter identifies a key role of SP1 in Kindlin-2 transcriptional regulation. Cell Mol Biol Lett 16: 638-651, 2011.

5. Xu Z, Gao J, Hong J and Ma YQ: Integrity of kindlin-2 FERM subdomains is required for supporting integrin activation. Biochem Biophys Res Commun 434: 382-387, 2013.

6. He Y, Esser P, Schacht V, Bruckner-Tuderman L and Has C: Role of kindlin-2 in fibroblast functions: implications for wound healing. J Invest Dermatol 131: 245-256, 2011.

7. Lai-Cheong JE, Parsons M and McGrath JA: The role of kindlins in cell biology and relevance to human disease. Int J Biochem Cell Biol 42: 595-603, 2010.

8. Montanez E, Ussar S, Schifferer M, Bösl M, Zent R, Moser M and Fässler R: Kindlin-2 controls bidirectional signaling of integrins. Genes Dev 22: 1325-1330, 2008.

9. Dowling JJ, Gibbs E, Russell M, Goldman D, Minarcik J, Golden JA and Feldman EL: Kindlin-2 is an essential component of intercalated discs and is required for vertebrate cardiac structure and function. Circ Res 102: 423-431, 2008

10. Pluskota E, Dowling JJ, Gordon N, Golden JA, Szpak D, West XZ, Nestor C, Ma YQ, Bialkowska K, Byzova T and Plow EF: The integrin coactivator kindlin-2 plays a critical role in angiogenesis in mice and zebrafish. Blood 117: 4978-4987, 2011.

11. Yu Y, Wu J, Wang Y, Zhao T, Ma B, Liu Y, Fang W, Zhu WG and Zhang $\mathrm{H}$ : Kindlin 2 forms a transcriptional complex with $\beta$-catenin and TCF4 to enhance Wnt signalling. EMBO Rep 13: 750-758, 2012.

12. Yu Y, Qi L, Wu J, Wang Y, Fang W and Zhang H: Kindlin 2 regulates myogenic related factor myogenin via a canonical Wnt signaling in myogenic differentiation. PLoS One 8: e63490, 2013.

13. van de Schans VA, Smits JF and Blankesteijn WM: The Wnt/frizzled pathway in cardiovascular development and disease: friend or foe? Eur J Pharmacol 585: 338-345, 2008.

14. Rao TP and Kühl M: An updated overview on Wnt signaling pathways: a prelude for more. Circ Res 106: 1798-1806, 2010.

15. Tsaousi A, Mill C and George SJ: The Wnt pathways in vascular disease: lessons from vascular development. Curr Opin Lipidol 22: 350-357, 2011 
16. Mill C and George SJ: Wnt signalling in smooth muscle cells and its role in cardiovascular disorders. Cardiovasc Res 95: 233-240, 2012.

17. Tsaousi A, Williams H, Lyon CA, Taylor V, Swain A, Johnson JL and George SJ: Wnt $4 / \beta$-catenin signaling induces VSMC proliferation and is associated with intimal thickening. Circ Res 108: 427-436, 2011.

18. Natarajan R, Pei H, Gu JL, Sarma JM and Nadler J: Evidence for 12-lipoxygenase induction in the vessel wall following balloon injury. Cardiovasc Res 41: 489-499, 1999.

19. Bao XL, Song H, Chen Z and Tang X: Wnt3a promotes epithelialmesenchymal transition, migration, and proliferation of lens epithelial cells. Mol Vis 18: 1983-1990, 2012.

20. Marchand A, Atassi F, Gaaya A, Leprince P, Le Feuvre C, Soubrier F, Lompré AM and Nadaud S: The Wnt/beta-catenin pathway is activated during advanced arterial aging in humans. Aging Cell 10: 220-232, 2011.

21. Harburger DS, Bouaouina M and Calderwood DA: Kindlin-1 and -2 directly bind the $\mathrm{C}$-terminal region of beta integrin cytoplasmic tails and exert integrin-specific activation effects. J Biol Chem 284: 11485-11497, 2009.

22. Malinin NL, Plow EF and Byzova TV: Kindlins in FERM adhesion. Blood 115: 4011-4017, 2010.

23. Meves A, Stremmel C, Gottschalk K and Fässler R: The kindlin protein family: new members to the club of focal adhesion proteins. Trends Cell Biol 19: 504-513, 2009.

24. Ussar S, Wang HV, Linder S, Fässler R and Moser M: The kindlins: subcellular localization and expression during murine development. Exp Cell Res 312: 3142-3151, 2006.

25. Ali RH and Khan AA: Tracing the evolution of FERM domain of Kindlins. Mol Phylogenet Evol 80: 193-204, 2014.

26. Dowling JJ, Vreede AP, Kim S, Golden J and Feldman EL: Kindlin-2 is required for myocyte elongation and is essential for myogenesis. BMC Cell Biol 9: 36, 2008.

27. Bandyopadhyay A, Rothschild G, Kim S, Calderwood DA and Raghavan S: Functional differences between kindlin-1 and kindlin-2 in keratinocytes. J Cell Sci 125: 2172-2184, 2012.
28. Qu H, Tu Y, Shi X, Larjava H, Saleem MA, Shattil SJ, Fukuda K, Qin J, Kretzler M and Wu C: Kindlin-2 regulates podocyte adhesion and fibronectin matrix deposition through interactions with phosphoinositides and integrins. J Cell Sci 124: 879-891, 2011.

29. Shi $X$ and Wu C: A suppressive role of mitogen inducible gene-2 in mesenchymal cancer cell invasion. Mol Cancer Res 6: 715-724, 2008.

30. Ma YQ, Qin J, Wu C and Plow EF: Kindlin-2 (Mig-2): a co-activator of beta3 integrins. J Cell Biol 181: 439-446, 2008 .

31. An Z, Dobra K, Lock JG, Stromblad S, Hjerpe A and Zhang H: Kindlin-2 is expressed in malignant mesothelioma and is required for tumor cell adhesion and migration. Int J Cancer 127: 1999-2008, 2010

32. Shen Z, Ye Y, Kauttu T, Seppänen H, Vainionpää S, Wang S, Mustonen $\mathrm{H}$ and Puolakkainen P: Novel focal adhesion protein kindlin-2 promotes the invasion of gastric cancer cells through phosphorylation of integrin $\beta 1$ and $\beta 3$. J Surg Oncol 108: 106-112, 2013.

33. Slater SC, Koutsouki E, Jackson CL, Bush RC, Angelini GD, Newby AC and George SJ: R-cadherin:beta-catenin complex and its association with vascular smooth muscle cell proliferation. Arterioscler Thromb Vasc Biol 24: 1204-1210, 2004.

34. Wang X, Xiao Y, Mou Y, Zhao Y, Blankesteijn WM and Hall JL: A role for the beta-catenin/T-cell factor signaling cascade in vascular remodeling. Circ Res 90: 340-347, 2002.

35. Yamada N, Noguchi S, Mori T, Naoe T, Maruo K and Akao Y: Tumor-suppressive microRNA-145 targets catenin $\delta-1$ to regulate Wnt/ $\beta$-catenin signaling in human colon cancer cells. Cancer Lett 335: 332-342, 2013.

36. Chen J, Zhang J, Xu L, Xu C, Chen S, Yang J and Jiang H: Inhibition of neointimal hyperplasia in the rat carotid artery injury model by a HMGB1 inhibitor. Atherosclerosis 224: 332-339, 2012. 